\title{
A new determinant expression for the weighted Bartholdi zeta function of a digraph
}

\author{
Iwao Sato*, Hideo Mitsuhasi \\ Oyama National College of Technology \\ Oyama, Tochigi 323-0806, Japan \\ isato@oyama-ct.ac.jp \\ Hideaki Morita \\ Division of System Engineering for Mathematics \\ Muroran Institute of Technology \\ Muroran, Hokkaido 050-8585, Japan
}

Submitted: Sep 10, 2013; Accepted: Jan 26, 2013; Published: Feb 5, 2013

Mathematical Subject Classifications: 05C50, 15A15

\begin{abstract}
We consider the weighted Bartholdi zeta function of a digraph $D$, and give a new determinant expression of it. Furthermore, we treat a weighted $L$-function of $D$, and give a new determinant expression of it. As a corollary, we present determinant expressions for the Bartholdi edge zeta functions of a graph and a digraph.
\end{abstract}

Key words: zeta function, digraph covering, $L$-function

\section{Introduction}

Zeta functions of graphs started from zeta functions of regular graphs by Ihara [7]. In [7], he showed that their reciprocals are explicit polynomials. A zeta function of a regular graph $G$ associated with a unitary representation of the fundamental group of $G$ was developed by Sunada [12,13]. Hashimoto [6] generalized Ihara's result on the zeta function of a regular graph to an irregular graph, and showed that its reciprocal is again a polynomial by a determinant containing the edge matrix. Bass [2] presented another determinant expression for the Ihara zeta function of an irregular graph by using its adjacency matrix.

Stark and Terras [11] gave an elementary proof of Bass' Theorem, and discussed three different zeta functions of any graph. Furthermore, various proofs of Bass' Theorem were given by Foata and Zeilberger [4], Kotani and Sunada [8].

\footnotetext{
*Supported by Grant-in-Aid for Science Research (C)
} 
For two variable zeta function of a graph, Bartholdi [1] defined and gave a determinant expression of the Bartholdi zeta function of a graph. Mizuno and Sato [9] presented a decomposition formula for the Bartholdi zeta function of a regular covering of a graph.

As a digraph version of the Bartholdi zeta function, Choe, Kwak, Park and Sato [3] defined the weighted Bartholdi zeta function of a digraph, and presented its determinant expression.

As a multi-variable zeta function of a graph, Stark and Terras [11] defined the edge zeta function of a graph. Watanabe and Fukumizu [14] presented a determinant expression for the edge zeta function of a graph $G$ with $n$ vertices by $n \times n$ matrices.

In this paper, we present a new determinant expression of the weighted Bartholdi zeta function of a digraph $D$ by using the method of Watanabe and Fukumizu [14]:

\section{Main Theorem.}

Let $D$ be a connected digraph with $n$ vertices and $m$ arcs, and let $\mathbf{W}=\mathbf{W}(D)$ be a weighted matrix of $D$. Then the reciprocal of the weighted Bartholdi zeta function of $D$ is given by

$$
\zeta(D, w, u, t)^{-1}=\operatorname{det}\left(\mathbf{I}_{n}+(1-u) t^{2} \tilde{\mathbf{D}}-t \tilde{\mathbf{A}}_{1}-t \tilde{\mathbf{A}}_{0}\right) \prod_{i=1}^{m_{1}}\left(1-w\left(f_{i}\right) w\left(f_{i}^{-1}\right)(1-u)^{2} t^{2}\right),
$$

where $\tilde{\mathbf{D}}, \tilde{\mathbf{A}}_{1}$ and $\tilde{\mathbf{A}}_{0}$ are defined in Section 3 , and $f_{1}^{ \pm 1}, \ldots, f_{m_{1}}^{ \pm 1}$ are symmetric arcs of $D$.

Furthermore, we present a new decomposition formula for the weighted Bartholdi zeta function of a group covering of $D$, and a new determinant expression for the weighted Bartholdi $L$-function of $D$.

\section{Preliminaries}

Graphs and digraphs treated here are finite. Let $G=(V(G), E(G))$ be a connected graph (possibly multiple edges and loops) with the set $V(G)$ of vertices and the set $E(G)$ of unoriented edges $u v$ joining two vertices $u$ and $v$. For $u v \in E(G)$, an arc $(u, v)$ is the oriented edge from $u$ to $v$. Set $D(G)=\{(u, v),(v, u) \mid u v \in E(G)\}$. For $e=(u, v) \in D(G)$, set $u=o(e)$ and $v=t(e)$. Furthermore, let $e^{-1}=(v, u)$ be the inverse of $e=(u, v)$.

A path $P$ of length $n$ in $G$ is a sequence $P=\left(e_{1}, \cdots, e_{n}\right)$ of $n$ arcs such that $e_{i} \in$ $D(G), t\left(e_{i}\right)=o\left(e_{i+1}\right)(1 \leqslant i \leqslant n-1)$, where indices are treated $\bmod n$. Set $|P|=n$, $o(P)=o\left(e_{1}\right)$ and $t(P)=t\left(e_{n}\right)$. Also, $P$ is called an $(o(P), t(P))$-path. We say that a path $P=\left(e_{1}, \cdots, e_{n}\right)$ has a backtracking or a bump at $t\left(e_{i}\right)$ if $e_{i+1}^{-1}=e_{i}$ for some $i(1 \leqslant i \leqslant n-1)$. A $(v, w)$-path is called a $v$-cycle (or $v$-closed path) if $v=w$.

We introduce an equivalence relation between cycles. Two cycles $C_{1}=\left(e_{1}, \cdots, e_{m}\right)$ and $C_{2}=\left(f_{1}, \cdots, f_{m}\right)$ are called equivalent if there exists $k$ such that $f_{j}=e_{j+k}$ for all $j$. The inverse cycle of $C$ is in general not equivalent to $C$. Let $[C]$ be the equivalence class which contains a cycle $C$. Let $B^{r}$ be the cycle obtained by going $r$ times around a cycle $B$. Such a cycle is called a power of $B$. A cycle $C$ is reduced if $C$ has no backtracking. Furthermore, a cycle $C$ is prime if it is not a power of a strictly smaller cycle. 
The Ihara zeta function of a graph $G$ is a function of $u \in \mathbf{C}$ with $|u|$ sufficiently small, defined by

$$
\mathbf{Z}(G, t)=\prod_{[C]}\left(1-t^{|C|}\right)^{-1}
$$

where $[C]$ runs over all equivalence classes of prime, reduced cycles of $G$ (see [7]).

Let $m$ be the number of edges of $G$. Furthermore, let two $m \times m$ matrices $\mathbf{B}=$ $\left(\mathbf{B}_{e, f}\right)_{e, f \in A(D)}$ and $\mathbf{J}_{0}=\left(\mathbf{J}_{e, f}\right)_{e, f \in A(D)}$ be defined as follows:

$$
\mathbf{B}_{e, f}=\left\{\begin{array}{ll}
1 & \text { if } t(e)=o(f), \\
0 & \text { otherwise }
\end{array}, \mathbf{J}_{e, f}=\left\{\begin{array}{ll}
1 & \text { if } f=e^{-1} \\
0 & \text { otherwise }
\end{array},\right.\right.
$$

Then $\mathbf{B}-\mathbf{J}_{0}$ is called the edge matrix of $G$.

Theorem 1 (Hashimoto; Bass). Let $G$ be a connected graph with $n$ vertices and $m$ edges. Then the reciprocal of the Ihara zeta function of $G$ is given by

$$
\mathbf{Z}(G, t)^{-1}=\operatorname{det}\left(\mathbf{I}_{2 m}-t\left(\mathbf{B}-\mathbf{J}_{0}\right)\right)=\left(1-t^{2}\right)^{m-n} \operatorname{det}\left(\mathbf{I}-t \mathbf{A}(G)+t^{2}(\mathbf{D}-\mathbf{I})\right),
$$

where $\mathbf{A}(G)$ is the adjacency matrix of $G$, and $\mathbf{D}=\left(d_{i j}\right)$ is the diagonal matrix with $d_{i i}=\operatorname{deg} v_{i}$ where $V(G)=\left\{v_{1}, \cdots, v_{n}\right\}$.

Then the Bartholdi zeta function of $G$ is defined by

$$
\zeta_{G}(u, t)=\zeta(G, u, t)=\prod_{[C]}\left(1-u^{c b c(C)} t^{|C|}\right)^{-1},
$$

where $[C]$ runs over all equivalence classes of prime cycles of $G($ see $[1])$.

Theorem 2 (Bartholdi). Let $G$ be a connected graph with $n$ vertices and $m$ unoriented edges. Then the reciprocal of the Bartholdi zeta function of $G$ is given by

$$
\begin{aligned}
\zeta(G, u, t)^{-1} & =\operatorname{det}\left(\mathbf{I}_{2 m}-t\left(\mathbf{B}-(1-u) \mathbf{J}_{0}\right)\right) \\
& =\left(1-(1-u)^{2} t^{2}\right)^{m-n} \operatorname{det}\left(\mathbf{I}-t \mathbf{A}(G)+(1-u)(\mathbf{D}-(1-u) \mathbf{I}) t^{2}\right) .
\end{aligned}
$$

In the case of $u=0$, Theorem 2 implies Theorem 1 .

We now state the weighted Bartholdi zeta function of a digraph. Let $D=(V(D), A(D))$ be a connected digraph with the set $V(D)$ of vertices and the set $A(D)$ of arcs. Furthermore, let $D$ have $n$ vertices $v_{1}, \ldots, v_{n}$ and $m$ arcs. Then we consider an $n \times n$ matrix $\mathbf{W}=\mathbf{W}(D)=\left(w_{i j}\right)_{1 \leqslant i, j \leqslant n}$ with $i j$ entry nonzero complex number $w_{i j}$ if $\left(v_{i}, v_{j}\right) \in A(D)$, and $w_{i j}=0$ otherwise. The matrix $\mathbf{W}=\mathbf{W}(D)$ is called the weighted matrix of $D$. Furthermore, let $w\left(v_{i}, v_{j}\right)=w_{i j}, v_{i}, v_{j} \in V(D)$ and $w(e)=w_{i j}, e=\left(v_{i}, v_{j}\right) \in A(D)$. For each path $P=\left(e_{1}, \cdots, e_{r}\right)$ of $G$, the norm $w(P)$ of $P$ is defined as follows: $w(P)=$ $w\left(e_{1}\right) \cdots w\left(e_{r}\right)$.

The cyclic bump count $\operatorname{cbc}(C)$ of a cycle $C=\left(e_{1}, \cdots, e_{n}\right)$ of $G$ is

$$
\operatorname{cbc}(C)=\left|\left\{i=1, \cdots, n \mid e_{i}=e_{i+1}^{-1}\right\}\right|,
$$


where $e_{n+1}=e_{1}$. Then the weighted Bartholdi zeta function of $D$ is a function of $u, t \in \mathbf{C}$ with $|u|,|t|$ sufficiently small, defined by

$$
\zeta(D, w, u, t)=\prod_{[C]}\left(1-w(C) u^{c b c(C)} t^{|C|}\right)^{-1},
$$

where $[C]$ runs over all equivalence classes of prime cycles of $D$.

If $w=1$, i.e., $w\left(v_{i}, v_{j}\right)=1$ for each $\left(v_{i}, v_{j}\right) \in A(D)$, then the weighted Bartholdi zeta function of $D$ is the Bartholdi zeta function of $D$. If $D=D_{G}$ is the symmetric digraph corresponding to a graph $G$, and $w=\mathbf{1}$, then the weighted Bartholdi zeta function of $D_{G}$ is the Bartholdi zeta function of $G$. If $D=D_{G}, w=\mathbf{1}$ and $u=0$, then the weighted Bartholdi zeta function of $G$ is the Ihara zeta function of $G$.

Two $m \times m$ matrices $\mathbf{B}_{w}=\left(\mathbf{B}_{e, f}^{w}\right)_{e, f \in A(D)}$ and $\mathbf{J}_{w}=\left(\mathbf{J}_{e, f}^{w}\right)_{e, f \in A(D)}$ are defined as follows:

$$
\mathbf{B}_{e, f}^{w}=\left\{\begin{array}{ll}
w(e) & \text { if } t(e)=o(f), \\
0 & \text { otherwise }
\end{array}, \mathbf{J}_{e, f}^{w}= \begin{cases}w(e) & \text { if } f=e^{-1} \\
0 & \text { otherwise }\end{cases}\right.
$$

Furthermore, we define two $n \times n$ matrices $\mathbf{W}_{1}=\mathbf{W}_{1}(D)=\left(a_{u v}\right)$ and $\mathbf{W}_{0}$ as follows:

$$
a_{u v}= \begin{cases}w(u, v) & \text { if } \operatorname{both}(u, v) \text { and }(v, u) \in A(D), \\ 0 & \text { otherwise }\end{cases}
$$

and

$$
\mathbf{W}_{0}=\mathbf{W}_{0}(D)=\mathbf{W}(D)-\mathbf{W}_{1} .
$$

Let an $n \times n$ matrix $\mathbf{S}=\left(s_{x y}\right)$ is the diagonal matrix defined by

$$
s_{x x}=\left|\left\{e \in A(D) \mid o(e)=x, e^{-1} \in A(D)\right\}\right| .
$$

Theorem 3 (Choe, Kwak, Park and Sato). Let $D$ be a connected digraph, and let $\mathbf{W}=$ $\mathbf{W}(D)$ be a weighted matrix of $D$. Furthermore, let $m_{1}=\left|\left\{e \in A(D) \mid e^{-1} \in A(D)\right\}\right| / 2$. Then the reciprocal of the weighted Bartholdi zeta function of $D$ is given by

$$
\zeta(D, w, u, t)^{-1}=\operatorname{det}\left(\mathbf{I}_{m}-\left(\mathbf{B}_{w}-(1-u) \mathbf{J}_{w}\right) t\right)
$$

where $n=|V(D)|$ and $m=|A(D)|$.

Furthermore, if $w\left(e^{-1}\right)=w(e)^{-1}$ for each $e \in A(D)$ such that $e^{-1} \in A(D)$, then

$$
\begin{aligned}
& \zeta(D, w, u, t)^{-1}=\left(1-(1-u)^{2} t^{2}\right)^{m_{1}-n} \\
\times & \operatorname{det}\left(\mathbf{I}_{n}-t \mathbf{W}_{1}(D)-\left(1-(1-u)^{2} t^{2}\right) t \mathbf{W}_{0}(D)+(1-u) t^{2}\left(\mathbf{S}-(1-u) \mathbf{I}_{n}\right)\right) .
\end{aligned}
$$

If $D=D_{G}, w=\mathbf{1}$ and $u=0$, then Theorem 2 implies Theorem 1 .

Now, we proceed to the edge zeta function of a graph $G$ with $m$ edges. Let $G$ be a connected graph and $D(G)=\left\{e_{1}, \ldots, e_{m}, e_{m+1}, \ldots, e_{2 m}\right\}\left(e_{m+i}=e_{i}^{-1}(1 \leqslant i \leqslant m)\right)$. We introduce $2 m$ variables $z_{1}, \ldots, z_{2 m}$, and set $g(C)=z_{i_{1}} \cdots z_{i_{k}}$ for each cycle $C=$ 
$\left(e_{i_{1}}, \ldots, e_{i_{k}}\right)$ of $G$. Set $z_{e_{i}}=z_{i}(1 \leqslant i \leqslant 2 m)$ and $\mathbf{z}=\left(z_{1}, \ldots, z_{2 m}\right)$. Then the edge zeta function $\zeta_{G}(\mathbf{z})$ of $G$ is defined by

$$
\zeta_{G}(\mathbf{z})=\prod_{[C]}(1-g(C))^{-1}
$$

where $[C]$ runs over all equivalence classes of prime, reduced cycles of $G$.

Theorem 4 (Stark and Terras). Let $G$ be a connected graph with $m$ edges. Then

$$
\zeta_{G}(\mathbf{z})^{-1}=\operatorname{det}\left(\mathbf{I}_{2 m}-\left(\mathbf{B}-\mathbf{J}_{0}\right) \mathbf{U}\right)
$$

where

$$
\mathbf{U}=\left[\begin{array}{cccccc}
z_{1} & & & & & 0 \\
& \ddots & & & & \\
& & z_{m} & & & \\
& & & z_{m+1} & & \\
& & & & \ddots & \\
0 & & & & & z_{2 m}
\end{array}\right]
$$

Let $G$ be a graph with $n$ vertices. Then we define an $n \times n$ matrix $\widehat{\mathbf{A}}=\left(a_{x y}\right)$ as follows:

$$
a_{x y}= \begin{cases}z_{(x, y)} /\left(1-z_{(x, y)} z_{(y, x)}\right) & \text { if }(x, y) \in D(G) \\ 0 & \text { otherwise }\end{cases}
$$

Furthermore, an $n \times n$ matrix $\widehat{\mathbf{D}}=\left(d_{x y}\right)$ is the diagonal matrix defined by

$$
d_{x x}=\sum_{o(e)=x} \frac{z_{e} z_{e}-1}{1-z_{e} z_{e^{-1}}} .
$$

Theorem 5 (Watanabe and Fukumizu). Let $G$ be a connected graph with $n$ vertices and $m$ edges. Then

$$
\zeta_{G}(\mathbf{z})^{-1}=\operatorname{det}\left(\mathbf{I}_{n}+\widehat{\mathbf{D}}-\widehat{\mathbf{A}}\right) \prod_{i=1}^{m}\left(1-z_{f_{i}} z_{f_{i}^{-1}}\right),
$$

where $D(G)=\left\{f_{1}, f_{1}^{-1}, \ldots, f_{m} f_{m}^{-1}\right\}$.

In Section 2, we present a new determinant expression of the weighted Bartholdi zeta function of a digraph $D$ by using the method of Watanabe and Fukumizu [14]. In Section 3 , we present a new decomposition formula for the weighted Bartholdi zeta function of a group covering of $D$. In Section 4, we present a new determinant expression for the weighted Bartholdi $L$-function of $D$. In Section 5 , we define the Bartholdi edge zeta functions of graphs and digraphs, and present their determinant expressions as corollaries of Theorem 6 . 


\section{Weighted Bartholdi zeta functions of digraphs}

We present a new determinant expression of the weighted Bartholdi zeta function of a digraph.

Let $D$ be a connected digraph with $n$ vertices $v_{1}, \cdots, v_{n}$ and $m$ arcs, and $\mathbf{W}=\mathbf{W}(D)$ a weighted matrix of $D$. Then we define two $n \times n$ matrices $\tilde{\mathbf{A}}_{1}=\tilde{\mathbf{A}}_{1}(D)=\left(a_{x y}\right)$ and $\tilde{\mathbf{A}}_{0}=\tilde{\mathbf{A}}_{0}(D)=\left(b_{x y}\right)$ as follows:

$$
a_{x y}= \begin{cases}w(x, y) /\left(1-w(x, y) w(y, x)(1-u)^{2} t^{2}\right) & \text { if both }(x, y) \text { and }(y, x) \in A(D), \\ 0 & \text { otherwise }\end{cases}
$$

and

$$
b_{x y}= \begin{cases}w(x, y) & \text { if }(x, y) \in A(D) \text { and }(y, x) \notin A(D), \\ 0 & \text { otherwise }\end{cases}
$$

Furthermore, an $n \times n$ matrix $\tilde{\mathbf{D}}=\tilde{\mathbf{D}}(D)=\left(d_{x y}\right)$ is the diagonal matrix defined by

$$
d_{x x}=\sum_{o(e)=x, e^{-1} \in A(D)} \frac{w(e) w\left(e^{-1}\right)}{1-w(e) w\left(e^{-1}\right)(1-u)^{2} t^{2}} .
$$

Let $\mathbf{M}_{1} \oplus \cdots \oplus \mathbf{M}_{s}$ be the block diagonal sum of square matrices $\mathbf{M}_{1}, \cdots, \mathbf{M}_{s}$. A new determinant expression for $\zeta(D, w, u, t)$ is given as follows:

Theorem 6. Let $D$ be a connected digraph, and let $\mathbf{W}=\mathbf{W}(D)$ be a weighted matrix of $D$. Then the reciprocal of the weighted Bartholdi zeta function of $D$ is given by

$$
\zeta(D, w, u, t)^{-1}=\operatorname{det}\left(\mathbf{I}_{n}+(1-u) t^{2} \tilde{\mathbf{D}}-t \tilde{\mathbf{A}}_{1}-t \tilde{\mathbf{A}}_{0}\right) \prod_{i=1}^{m_{1}}\left(1-w\left(f_{i}\right) w\left(f_{i}^{-1}\right)(1-u)^{2} t^{2}\right),
$$

where $n=|V(D)|, m=|A(D)|$ and $f_{1}^{ \pm 1}, \ldots, f_{m_{1}}^{ \pm 1}$ are symmetric arcs of $D$.

Proof. Let $V(D)=\left\{v_{1}, \cdots, v_{n}\right\}$ and, let $A(D)=\left\{e_{1}, \cdots, e_{m_{0}}, f_{1}, \cdots, f_{m_{1}}, f_{1}^{-1}, \cdots\right.$, $\left.f_{m_{1}}^{-1}\right\}$ such that $e_{i}^{-1} \notin A(D)\left(1 \leqslant i \leqslant m_{0}\right)$. Note that $m=m_{0}+2 m_{1}$.

Arrange arcs of $D$ as follows:

$$
e_{1}, \cdots, e_{m_{0}}, f_{1}, f_{1}^{-1}, \cdots, f_{m_{1}}, f_{m_{1}}^{-1}
$$

Let

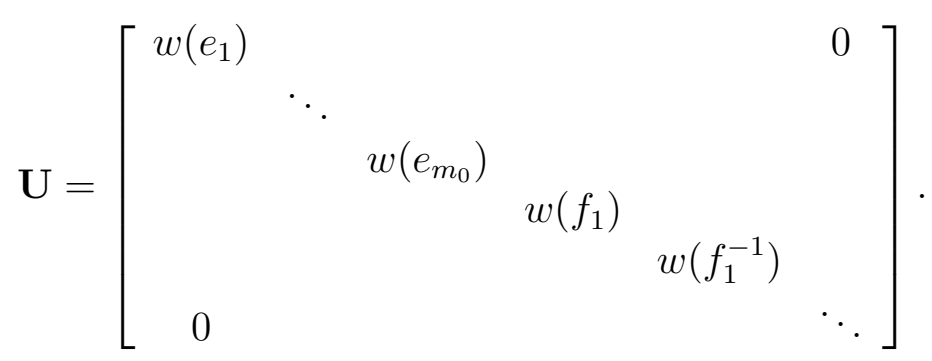

Then we have

$$
\mathbf{U B}=\mathbf{B}_{w} \text { and } \mathbf{U} \mathbf{J}_{0}=\mathbf{J}_{w}
$$


Thus,

$$
\mathbf{B}_{w}-(1-u) \mathbf{J}_{w}=\mathbf{U}\left(\mathbf{B}-(1-u) \mathbf{J}_{0}\right) .
$$

By Theorem 2, it follows that

$$
\zeta(D, w, u, t)^{-1}=\operatorname{det}\left(\mathbf{I}_{m}-t \mathbf{U}\left(\mathbf{B}-(1-u) \mathbf{J}_{0}\right)\right) .
$$

Now, let $\mathbf{K}=\left(\mathbf{K}_{e v}\right)_{e \in A(D) ; v \in V(D)}$ be the $m \times n$ matrix defined as follows:

$$
\mathbf{K}_{e v}:= \begin{cases}1 & \text { if } o(e)=v \\ 0 & \text { otherwise }\end{cases}
$$

Furthermore, we define the $m \times n$ matrix $\mathbf{L}=\left(\mathbf{L}_{e v}\right)_{e \in A(D) ; v \in V(D)}$ as follows:

$$
\mathbf{L}_{e v}:= \begin{cases}1 & \text { if } t(e)=v \\ 0 & \text { otherwise. }\end{cases}
$$

Then we have

$$
\mathbf{L}^{t} \mathbf{K}=\mathbf{B}
$$

Thus,

$$
\begin{aligned}
& \operatorname{det}\left(\mathbf{I}_{m}-t \mathbf{U}\left(\mathbf{B}-(1-u) \mathbf{J}_{0}\right)\right) \\
= & \operatorname{det}\left(\mathbf{I}_{m}-t \mathbf{U}\left(\mathbf{L}^{t} \mathbf{K}-(1-u) \mathbf{J}_{0}\right)\right)=\operatorname{det}\left(\mathbf{I}_{m}-t \mathbf{U} \mathbf{L}^{t} \mathbf{K}+(1-u) t \mathbf{U} \mathbf{J}_{0}\right) .
\end{aligned}
$$

But, we have

$$
\mathbf{I}_{m}+(1-u) t \mathbf{U} \mathbf{J}_{0}=\mathbf{I}_{m_{0}} \oplus\left(\oplus_{j=1}^{m_{1}}\left[\begin{array}{cc}
1 & (1-u) t w\left(f_{j}\right) \\
(1-u) t w\left(f_{j}^{-1}\right) & 1
\end{array}\right]\right) .
$$

Since $|u|,|t|$ are sufficiently small, we have

$$
\operatorname{det}\left(\left[\begin{array}{cc}
1 & (1-u) t w\left(f_{j}\right) \\
(1-u) t w\left(f_{j}^{-1}\right) & 1
\end{array}\right]\right)=1-(1-u)^{2} t^{2} w\left(f_{j}\right) w\left(f_{j}^{-1}\right) \neq 0\left(1 \leqslant j \leqslant m_{1}\right) .
$$

Thus, $\mathbf{I}_{m}+(1-u) t \mathbf{U} \mathbf{J}_{0}$ is invertible. Therefore,

$$
\begin{aligned}
& \operatorname{det}\left(\mathbf{I}_{m}-t \mathbf{U}\left(\mathbf{B}-(1-u) \mathbf{J}_{0}\right)\right) \\
= & \operatorname{det}\left(\mathbf{I}_{m}-t \mathbf{U} \mathbf{L}^{t} \mathbf{K}\left(\mathbf{I}_{m}+(1-u) t \mathbf{U} \mathbf{J}_{0}\right)^{-1}\right) \operatorname{det}\left(\mathbf{I}_{m}+(1-u) t \mathbf{U} \mathbf{J}_{0}\right) .
\end{aligned}
$$

But, if $\mathbf{A}$ and $\mathbf{B}$ are a $m \times n$ and $n \times m$ matrices, respectively, then we have

$$
\operatorname{det}\left(\mathbf{I}_{m}-\mathbf{A B}\right)=\operatorname{det}\left(\mathbf{I}_{n}-\mathbf{B A}\right) .
$$

Thus, we have

$$
\begin{aligned}
& \operatorname{det}\left(\mathbf{I}_{m}-t \mathbf{U}\left(\mathbf{B}-(1-u) \mathbf{J}_{0}\right)\right) \\
= & \operatorname{det}\left(\mathbf{I}_{n}-t{ }^{t} \mathbf{K}\left(\mathbf{I}_{m}+(1-u) t \mathbf{U} \mathbf{J}_{0}\right)^{-1} \mathbf{U L}\right) \operatorname{det}\left(\mathbf{I}_{m}+(1-u) t \mathbf{U} \mathbf{J}_{0}\right) .
\end{aligned}
$$


Next, we have

$$
\operatorname{det}\left(\mathbf{I}_{m}+(1-u) t \mathbf{U} \mathbf{J}_{0}\right)=\prod_{i=1}^{m_{1}}\left(1-w\left(f_{i}\right) w\left(f_{i}^{-1}\right)(1-u)^{2} t^{2}\right) .
$$

Furthermore, the $m \times n$ matrix $\mathbf{U L}=\left(c_{e v}\right)_{e \in A(D) ; v \in V(D)}$ is given as follows:

$$
c_{e v}:= \begin{cases}w(e) & \text { if } t(e)=v \\ 0 & \text { otherwise }\end{cases}
$$

But, we have

$$
\left(\mathbf{I}_{m}+(1-u) t \mathbf{U} \mathbf{J}_{0}\right)^{-1}=\mathbf{I}_{m_{0}} \oplus\left(\oplus_{j=1}^{m_{1}}\left[\begin{array}{cc}
1 / x_{j} & -(1-u) t w\left(f_{j}\right) / x_{j} \\
-(1-u) t w\left(f_{j}^{-1}\right) / x_{j} & 1 / x_{j}
\end{array}\right]\right),
$$

where $x_{i}=1-w\left(f_{i}\right) w\left(f_{i}^{-1}\right)(1-u)^{2} t^{2}\left(1 \leqslant i \leqslant m_{1}\right)$.

Now, for a symmetric arc $(x, y) \in A(D)$,

$$
\left({ }^{t} \mathbf{K}\left(\mathbf{I}_{m}+(1-u) t \mathbf{U} \mathbf{J}_{0}\right)^{-1} \mathbf{U L}\right)_{x y}=w(x, y) /\left(1-w(x, y) w(y, x)(1-u)^{2} t^{2}\right) .
$$

For a nonsymmetric arc $(x, y) \in A(D)$,

$$
\left({ }^{t} \mathbf{K}\left(\mathbf{I}_{m}+(1-u) t \mathbf{U} \mathbf{J}_{0}\right)^{-1} \mathbf{U L}\right)_{x y}=w(x, y) .
$$

Furthermore, if $x=y$, then

$$
\left({ }^{t} \mathbf{K}\left(\mathbf{I}_{m}+(1-u) t \mathbf{U} \mathbf{J}_{0}\right)^{-1} \mathbf{U L}\right)_{x x}=-\sum_{o(e)=x, e^{-1} \in A(D)} \frac{(1-u) t w(e) w\left(e^{-1}\right)}{1-w(e) w\left(e^{-1}\right)(1-u)^{2} t^{2}} .
$$

Thus,

$$
\operatorname{det}\left(\mathbf{I}_{n}-t^{t} \mathbf{K}\left(\mathbf{I}_{m}+(1-u) t \mathbf{U} \mathbf{J}_{0}\right)^{-1} \mathbf{U L}\right)=\operatorname{det}\left(\mathbf{I}_{n}+(1-u) t^{2} \tilde{\mathbf{D}}-t \tilde{\mathbf{A}}_{1}-t \tilde{\mathbf{A}}_{0}\right) .
$$

Therefore, it follows that

$$
\zeta(D, w, u, t)^{-1}=\operatorname{det}\left(\mathbf{I}_{n}+(1-u) t^{2} \tilde{\mathbf{D}}-t \tilde{\mathbf{A}}_{1}-t \tilde{\mathbf{A}}_{0}\right) \prod_{i=1}^{m_{1}}\left(1-w\left(f_{i}\right) w\left(f_{i}^{-1}\right)(1-u)^{2} t^{2}\right) .
$$

By Theorem 5, we obtain the second identity of Theorem 2.

Corollary 1 (Choe, Kwak, Park and Sato). Let $D$ be a connected digraph, and let $\mathbf{W}=$ $\mathbf{W}(D)$ be a weighted matrix of $D$. Furthermore, assume that $w\left(e^{-1}\right)=w(e)^{-1}$ for each $e \in A(D)$ such that $e^{-1} \in A(D)$. Then the reciprocal of the weighted Bartholdi zeta function of $D$ is given by

$$
\begin{aligned}
& \zeta(D, w, u, t)^{-1}=\left(1-(1-u)^{2} t^{2}\right)^{m_{1}-n} \\
\times & \operatorname{det}\left(\mathbf{I}_{n}-t \mathbf{W}_{1}(D)-\left(1-(1-u)^{2} t^{2}\right) t \mathbf{W}_{0}(D)+(1-u) t^{2}\left(\mathbf{S}-(1-u) \mathbf{I}_{n}\right)\right) .
\end{aligned}
$$

where $n=|V(D)|$ and $m=|A(D)|$. 
Proof. Since $w\left(e^{-1}\right)=w(e)^{-1}$ for each symmetric arc $e \in A(D)$, we have $w\left(e^{-1}\right)$ $w(e)^{-1}=1$. Then we have

$$
\tilde{\mathbf{D}}=\frac{1}{1-(1-u)^{2} t^{2}} \mathbf{S}, \quad \tilde{\mathbf{A}}_{1}=\frac{1}{1-(1-u)^{2} t^{2}} \mathbf{W}_{1}(D) .
$$

Furthermore, $\tilde{\mathbf{A}}_{0}=\mathbf{W}_{0}(D)$. Thus,

$$
\begin{aligned}
\zeta(D, w, u, t)^{-1}= & \left(1-(1-u)^{2} t^{2}\right)^{m_{1}} \operatorname{det}\left(\mathbf{I}_{n}-t /\left(1-(1-u)^{2} t^{2}\right) \mathbf{W}_{1}(D)\right. \\
& \left.-t \mathbf{W}_{0}(D)+(1-u) t^{2} /\left(1-(1-u)^{2} t^{2}\right) \mathbf{S}\right) \\
= & \left(1-(1-u)^{2} t^{2}\right)^{m_{1}-n} \operatorname{det}\left(\mathbf{I}_{n}-t \mathbf{W}_{1}(D)-\left(1-(1-u)^{2} t^{2}\right) t \mathbf{W}_{0}(D)\right. \\
& \left.+(1-u) t^{2}\left(\mathbf{S}-(1-u) \mathbf{I}_{n}\right)\right) .
\end{aligned}
$$

\section{Weighted Bartholdi zeta functions of group cover- ings of digraphs}

We can generalize the notion of a $\Gamma$-covering of a graph to a simple digraph. Let $D$ be a connected digraph and $\Gamma$ a finite group. Then a mapping $\alpha: A(D) \longrightarrow \Gamma$ is called a pseudo ordinary voltage assignment if $\alpha(v, u)=\alpha(u, v)^{-1}$ for each $(u, v) \in A(D)$ such that $(v, u) \in A(D)$. The pair $(D, \alpha)$ is called an ordinary voltage digraph. The derived digraph $D^{\alpha}$ of the ordinary voltage digraph $(D, \alpha)$ is defined as follows: $V\left(D^{\alpha}\right)=V(D) \times \Gamma$ and $((u, h),(v, k)) \in A\left(D^{\alpha}\right)$ if and only if $(u, v) \in A(D)$ and $k=h \alpha(u, v)$. The digraph $D^{\alpha}$ is called a $\Gamma$-covering of $D$. Note that a $\Gamma$-covering of the symmetric digraph corresponding to a graph $G$ is a $\Gamma$-covering of $G$ (see [5]).

Let $D$ be a connected digraph, $\Gamma$ a finite group and $\alpha: A(D) \longrightarrow \Gamma$ a pseudo ordinary voltage assignment. In the $\Gamma$-covering $D^{\alpha}$, set $v_{g}=(v, g)$ and $e_{g}=(e, g)$, where $v \in$ $V(D), e \in A(D), g \in \Gamma$. For $e=(u, v) \in A(D)$, the arc $e_{g}$ emanates from $u_{g}$ and terminates at $v_{g \alpha(e)}$.

Let $\mathbf{W}=\mathbf{W}(D)$ be a weighted matrix of $D$. Then we define the weighted matrix $\tilde{\mathbf{W}}=\mathbf{W}\left(D^{\alpha}\right)=\left(\tilde{w}\left(u_{g}, v_{h}\right)\right)$ of $D^{\alpha}$ derived from $\mathbf{W}$ as follows:

$$
\tilde{w}\left(u_{g}, v_{h}\right):= \begin{cases}w(u, v) & \text { if }(u, v) \in A(D) \text { and } h=g \alpha(u, v), \\ 0 & \text { otherwise. }\end{cases}
$$

If $\mathbf{M}_{1}=\mathbf{M}_{2}=\cdots=\mathbf{M}_{s}=\mathbf{M}$, then we write $s \circ \mathbf{M}=\mathbf{M}_{1} \oplus \cdots \oplus \mathbf{M}_{s}$. The Kronecker product $\mathbf{A} \otimes \mathbf{B}$ of matrices $\mathbf{A}$ and $\mathbf{B}$ is considered as the matrix $\mathbf{A}$ having the element $a_{i j}$ replaced by the matrix $a_{i j} \mathbf{B}$.

Theorem 7. Let $D$ be a connected digraph with $n$ vertices and $m$ arcs, $\Gamma$ a finite group, $\alpha: A(D) \longrightarrow \Gamma$ a pseudo ordinary voltage assignment and $\mathbf{W}=\mathbf{W}(D)$ a weighted 
matrix of D. Set $m_{1}=\left|\left\{e \in A(D) \mid e^{-1} \in A(D)\right\}\right| / 2$ and $|\Gamma|=r$. Furthermore, let $\rho_{1}=1, \rho_{2}, \cdots, \rho_{k}$ be the irreducible representations of $\Gamma$, and $d_{i}$ the degree of $\rho_{i}$ for each $i$, where $d_{1}=1$. For $g \in \Gamma$, the matrix $\mathbf{A}_{1, g}=\left(a_{x y}^{(g)}\right)$ is defined as follows:

$a_{x y}^{(g)}:= \begin{cases}w(x, y) /\left(1-w(x, y) w(y, x)(1-u)^{2} t^{2}\right) & \text { if }(x, y),(y, x) \in A(D) \text { and } \alpha(x, y)=g \\ 0 & \text { otherwise. }\end{cases}$

Furthermore, the matrix $\mathbf{A}_{0, g}=\left(b_{x y}^{(g)}\right)$ is defined as follows:

$$
b_{x y}^{(g)}:= \begin{cases}w(x, y) & \text { if }(x, y) \in A(D),(y, x) \notin A(D) \text { and } \alpha(x, y)=g, \\ 0 & \text { otherwise. }\end{cases}
$$

Suppose that the $\Gamma$-covering $D^{\alpha}$ of $D$ is connected. Then the reciprocal of the weighted Bartholdi zeta function of $D^{\alpha}$ is

$$
\begin{gathered}
\zeta\left(D^{\alpha}, \tilde{w}, u, t\right)^{-1}=\prod_{i=1}^{m_{1}}\left(1-w\left(f_{i}\right) w\left(f_{i}^{-1}\right)(1-u)^{2} t^{2}\right)^{r} \\
\times \prod_{i=1}^{k}\left\{\operatorname{det}\left(\mathbf{I}_{n d_{i}}-t \sum_{h \in \Gamma} \rho_{i}(h) \bigotimes \mathbf{A}_{1, h}-t \sum_{h \in \Gamma} \rho_{i}(h) \bigotimes \mathbf{A}_{0, h}+(1-u) t^{2}\left(\mathbf{I}_{d_{i}} \bigotimes \tilde{\mathbf{D}}(D)\right)\right)\right\}^{d_{i}},
\end{gathered}
$$

where $f_{1}^{ \pm 1}, \ldots, f_{m_{1}}^{ \pm 1}$ are symmetric arcs of $D$.

Proof . Let $V(D)=\left\{v_{1}, \cdots, v_{n}\right\}$ and $\Gamma=\left\{1=g_{1}, g_{2}, \cdots, g_{r}\right\}$. Arrange vertices of $D^{\alpha}$ in $n$ blocks: $\left(v_{1}, 1\right), \cdots,\left(v_{n}, 1\right) ;\left(v_{1}, g_{2}\right), \cdots,\left(v_{n}, g_{2}\right) ; \cdots ;\left(v_{1}, g_{r}\right), \cdots,\left(v_{n}, g_{r}\right)$. We consider the three matrices $\tilde{\mathbf{A}}_{1}\left(D^{\alpha}\right), \tilde{\mathbf{W}}_{0}\left(D^{\alpha}\right)$ and $\tilde{\mathbf{D}}\left(D^{\alpha}\right)$ under this order. By Theorem 5 , we have

$$
\begin{aligned}
\zeta\left(D^{\alpha}, \tilde{w}, u, t\right)^{-1}= & \operatorname{det}\left(\mathbf{I}_{\nu m}-t \tilde{\mathbf{A}}_{1}\left(D^{\alpha}\right)-t \tilde{\mathbf{A}}_{0}\left(D^{\alpha}\right)+(1-u) t^{2} \tilde{\mathbf{D}}\left(D^{\alpha}\right)\right) \\
& \cdot \prod_{i=1}^{m_{1}}\left(1-w\left(f_{i}\right) w\left(f_{i}^{-1}\right)(1-u)^{2} t^{2}\right)^{r} .
\end{aligned}
$$

For $h \in \Gamma$, the matrix $\mathbf{P}_{h}=\left(p_{i j}^{(h)}\right)$ is defined as follows:

$$
p_{i j}^{(h)}= \begin{cases}1 & \text { if } g_{i} h=g_{j} \\ 0 & \text { otherwise }\end{cases}
$$

Suppose that $p_{i j}^{(h)}=1$, i.e., $g_{j}=g_{i} h$. Then $\left(\left(u, g_{i}\right),\left(v, g_{j}\right)\right) \in A\left(D^{\alpha}\right)$ if and only if $(u, v) \in A(D)$ and $g_{j}=g_{i} \alpha(u, v)$, i.e., $\alpha(u, v)=g_{i}^{-1} g_{j}=g_{i}^{-1} g_{i} h=h$. Thus we have

$$
\tilde{\mathbf{A}}_{0}\left(D^{\alpha}\right)=\sum_{h \in \Gamma} \mathbf{P}_{h} \bigotimes \mathbf{A}_{0, h} \text { and } \tilde{\mathbf{A}}_{1}\left(D^{\alpha}\right)=\sum_{h \in \Gamma} \mathbf{P}_{h} \bigotimes \mathbf{A}_{1, h} .
$$

Let $\rho$ be the right regular representation of $\Gamma$. Furthermore, let $\rho_{1}=1, \rho_{2}, \cdots, \rho_{k}$ be the irreducible representations of $\Gamma$, and $d_{i}$ the degree of $\rho_{i}$ for each $i$, where $d_{1}=1$. Then 
we have $\rho(h)=\mathbf{P}_{h}$ for $h \in \Gamma$. Furthermore, there exists a nonsingular matrix $\mathbf{P}$ such that $\mathbf{P}^{-1} \rho(h) \mathbf{P}=(1) \oplus d_{2} \circ \rho_{2}(h) \oplus \cdots \oplus d_{k} \circ \rho_{k}(h)$ for each $h \in \Gamma$ (see [10]). Putting $\mathbf{B}=\left(\mathbf{P}^{-1} \otimes \mathbf{I}_{n}\right)\left(\tilde{\mathbf{A}}_{1}\left(D^{\alpha}\right)+\tilde{\mathbf{A}}_{0}\left(D^{\alpha}\right)\right)\left(\mathbf{P} \otimes \mathbf{I}_{n}\right)$, we have

$$
\mathbf{B}=\sum_{h \in \Gamma}\left\{(1) \oplus d_{2} \circ \rho_{2}(h) \oplus \cdots \oplus d_{k} \circ \rho_{k}(h)\right\} \bigotimes\left(\mathbf{A}_{1, h}+\mathbf{A}_{0, h}\right) .
$$

Note that $\tilde{\mathbf{A}}_{i}(D)=\sum_{h \in \Gamma} \mathbf{A}_{i, h}(i=0,1)$ and $1+d_{2}^{2}+\cdots+d_{k}^{2}=r$. Therefore it follows that

$$
\begin{gathered}
\zeta\left(D^{\alpha}, \tilde{w}, u, t\right)^{-1}=\prod_{j=1}^{m_{1}}\left(1-w\left(f_{j}\right) w\left(f_{j}^{-1}\right)(1-u)^{2} t^{2}\right)^{r} \\
\times \prod_{i=1}^{k} \operatorname{det}\left(\mathbf{I}_{n d_{i}}-t \sum_{h \in \Gamma} \rho_{i}(h) \bigotimes \mathbf{A}_{1, h}-t \sum_{h \in \Gamma} \rho_{i}(h) \bigotimes \mathbf{A}_{0, h}+(1-u) t^{2}\left(\mathbf{I}_{d_{i}} \bigotimes \tilde{\mathbf{D}}(D)\right)\right)^{d_{i}} .
\end{gathered}
$$

\section{$5 \quad L$-functions of digraphs}

Let $D$ be a connected digraph with $m$ arcs, $\Gamma$ a finite group, $\alpha: A(D) \longrightarrow \Gamma$ a pseudo ordinary voltage assignment and $\mathbf{W}=\mathbf{W}(D)$ a weighted matrix of $D$. For each path $P=$ $\left(e_{1}, \cdots, e_{l}\right)$ of $D$, set $\alpha(P)=\alpha\left(e_{1}\right) \cdots \alpha\left(e_{l}\right)$ and $w(P)=w\left(e_{1}\right) \cdots w\left(e_{l}\right)$. Furthermore, let $\rho$ be a representation of $\Gamma$ and $d$ its degree.

The weighted Bartholdi L-function of $D$ associated with $\rho$ and $\alpha$ is defined by

$$
\zeta_{D}(w, u, t, \rho, \alpha)=\prod_{[C]} \operatorname{det}\left(\mathbf{I}_{d}-w(C) \rho(\alpha(C)) u^{c b c(C)} t^{|C|}\right)^{-1}
$$

where $[C]$ runs over all equivalence classes of prime cycles of $D$.

Two $m d \times m d$ matrices $\mathbf{B}_{w}^{\rho}=\left(\mathbf{B}_{e, f}\right)_{e, f \in A(D)}$ and $\mathbf{J}_{w}^{\rho}=\left(\mathbf{J}_{e, f}\right)_{e, f \in A(D)}$ are defined as follows:

$$
\mathbf{B}_{e, f}=\left\{\begin{array}{ll}
w(e) \rho(\alpha(e)) & \text { if } t(e)=o(f), \\
\mathbf{0}_{d} & \text { otherwise }
\end{array}, \mathbf{J}_{e, f}= \begin{cases}w(e) \rho(\alpha(e)) & \text { if } f=e^{-1} \\
\mathbf{0}_{d} & \text { otherwise }\end{cases}\right.
$$

A determinant expression for the weighted Bartholdi $L$-function of $D$ associated with $\rho$ and $\alpha$ was given by Choe, Kwak, Park and Sato [3]. Let $1 \leqslant i, j \leqslant n$. Then the $(i, j)-$ block $\mathbf{F}_{i j}$ of a $d n \times d n$ matrix $\mathbf{F}$ is the submatrix of $\mathbf{F}$ consisting of $d(i-1)+1, \ldots, d i$ rows and $d(j-1)+1, \ldots, d j$ columns.

Theorem 8 (Choe, Kwak, Park and Sato). Let $D$ be a connected digraph with $m$ arcs, $\Gamma$ a finite group, $\alpha: A(D) \longrightarrow \Gamma$ a pseudo ordinary voltage assignment and $\mathbf{W}=\mathbf{W}(D)$ a weighted matrix of $D$. Furthermore, let $\rho$ be a representation of $\Gamma$, and $d$ the degree of $\rho$. Then the reciprocal of the weighted Bartholdi L-function of $D$ associated with $\rho$ and $\alpha$ is

$$
\zeta_{D}(w, u, t, \rho, \alpha)^{-1}=\operatorname{det}\left(\mathbf{I}_{m d}-\left(\mathbf{B}_{w}^{\rho}-(1-u) \mathbf{J}_{w}^{\rho}\right) t\right) .
$$


A new determinant expression for the weighted Bartholdi $L$-function of $D$ associated with $\rho$ and $\alpha$ is given as follows:

Theorem 9. Let $D$ be a connected digraph, and let $\mathbf{W}=\mathbf{W}(D)$ be a weighted matrix of $D$. Then the reciprocal of the weighted Bartholdi L-function of $D$ is given by

$$
\begin{gathered}
\zeta_{D}(w, u, t, \rho, \alpha)^{-1}=\prod_{i=1}^{m_{1}}\left(1-w\left(f_{i}\right) w\left(f_{i}^{-1}\right)(1-u)^{2} t^{2}\right)^{d} \\
\times \operatorname{det}\left(\mathbf{I}_{n d}+(1-u) t^{2} \mathbf{I}_{d} \bigotimes \tilde{\mathbf{D}}(D)-t \sum_{g \in \Gamma} \rho(g) \bigotimes \mathbf{A}_{1, g}-t \sum_{g \in \Gamma} \rho(g) \bigotimes \mathbf{A}_{0, g}\right),
\end{gathered}
$$

where $n=|V(D)|, m=|A(D)|$ and $f_{1}^{ \pm 1}, \ldots, f_{m_{1}}^{ \pm 1}$ are symmetric arcs of $D$.

Proof. Let $V(D)=\left\{v_{1}, \cdots, v_{n}\right\}$ and, let $A(D)=\left\{e_{1}, \cdots, e_{m_{0}}, f_{1}, \cdots, f_{m_{1}}, f_{1}^{-1}, \cdots\right.$, $\left.f_{m_{1}}^{-1}\right\}$ such that $e_{i}^{-1} \notin A(D)\left(1 \leqslant i \leqslant m_{0}\right)$. Note that $m=m_{0}+2 m_{1}$.

Arrange arcs of $D$ as follows:

$$
e_{1}, \cdots, e_{m_{0}}, f_{1}, f_{1}^{-1}, \cdots, f_{m_{1}}, f_{m_{1}}^{-1}
$$

Let

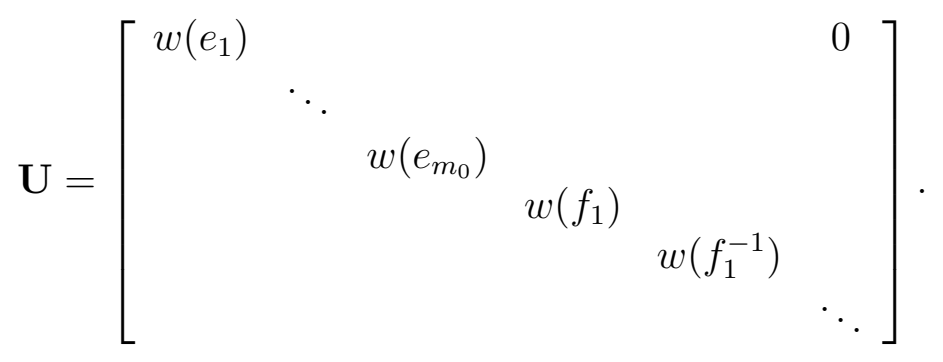

Furthermore, let two $m d \times m d$ matrices $\mathbf{B}_{\rho}=\left(\mathbf{B}_{e, f}^{\rho}\right)_{e, f \in A(D)}$ and $\mathbf{J}_{\rho}=\left(\mathbf{J}_{e, f}^{\rho}\right)_{e, f \in A(D)}$ be defined as follows:

$$
\mathbf{B}_{e, f}^{\rho}=\left\{\begin{array}{ll}
\rho(\alpha(e)) & \text { if } t(e)=o(f), \\
\mathbf{0}_{d} & \text { otherwise }
\end{array}, \mathbf{J}_{e, f}^{\rho}= \begin{cases}\rho(\alpha(e)) & \text { if } f=e^{-1} \\
\mathbf{0}_{d} & \text { otherwise. }\end{cases}\right.
$$

Then we have

$$
\left(\mathbf{U} \bigotimes \mathbf{I}_{d}\right) \mathbf{B}_{\rho}=\mathbf{B}_{w}^{\rho} \text { and }\left(\mathbf{U} \bigotimes \mathbf{I}_{d}\right) \mathbf{J}_{\rho}=\mathbf{J}_{w}^{\rho}
$$

Thus,

$$
\mathbf{B}_{w}^{\rho}-(1-u) \mathbf{J}_{w}^{\rho}=\left(\mathbf{U} \bigotimes \mathbf{I}_{d}\right)\left(\mathbf{B}_{\rho}-(1-u) \mathbf{J}_{\rho}\right) .
$$

By Theorem 7, it follows that

$$
\zeta_{D}(w, u, t, \rho, \alpha)^{-1}=\operatorname{det}\left(\mathbf{I}_{m d}-t\left(\mathbf{U} \bigotimes \mathbf{I}_{d}\right)\left(\mathbf{B}_{\rho}-(1-u) \mathbf{J}_{\rho}\right)\right) .
$$

Now, let $\mathbf{K}=\left(\mathbf{K}_{e v}\right)_{e \in A(D) ; v \in V(D)}$ be the $m d \times n d$ matrix defined as follows:

$$
\mathbf{K}_{e v}:= \begin{cases}\mathbf{I}_{d} & \text { if } o(e)=v \\ \mathbf{0}_{d} & \text { otherwise }\end{cases}
$$


Furthermore, we define the $m d \times n d$ matrix $\mathbf{L}=\left(\mathbf{L}_{e v}\right)_{e \in A(D) ; v \in V(D)}$ as follows:

$$
\mathbf{L}_{e v}:= \begin{cases}\rho(\alpha(e)) & \text { if } t(e)=v, \\ \mathbf{0}_{d} & \text { otherwise. }\end{cases}
$$

Set $\mathbf{U}_{d}=\mathbf{U} \otimes \mathbf{I}_{d}$. Then we have

$$
\mathbf{L}^{t} \mathbf{K}=\mathbf{B}_{\rho}
$$

Thus,

$$
\begin{aligned}
& \operatorname{det}\left(\mathbf{I}_{m d}-t \mathbf{U}_{d}\left(\mathbf{B}_{\rho}-(1-u) \mathbf{J}_{\rho}\right)\right) \\
= & \operatorname{det}\left(\mathbf{I}_{m d}-t \mathbf{U}_{d}\left(\mathbf{L}^{t} \mathbf{K}-(1-u) \mathbf{J}_{\rho}\right)\right)=\operatorname{det}\left(\mathbf{I}_{m d}-t \mathbf{U}_{d} \mathbf{L}^{t} \mathbf{K}+(1-u) t \mathbf{U}_{d} \mathbf{J}_{\rho}\right) .
\end{aligned}
$$

But, we have

$$
\begin{gathered}
\mathbf{I}_{m d}+(1-u) t \mathbf{U}_{d} \mathbf{J}_{\rho} \\
=\mathbf{I}_{m_{0} d} \oplus\left(\oplus_{j=1}^{m_{1}}\left[\begin{array}{cc}
\mathbf{I}_{d} & (1-u) t w\left(f_{j}\right) \rho\left(\alpha\left(f_{j}\right)\right) \\
(1-u) t w\left(f_{j}^{-1}\right) \rho\left(\alpha\left(f_{j}^{-1}\right)\right) & \mathbf{I}_{d}
\end{array}\right]\right) .
\end{gathered}
$$

Since $|u|,|t|$ are sufficiently small, we have

$$
\begin{gathered}
\operatorname{det}\left(\left[\begin{array}{cc}
\mathbf{I}_{d} & (1-u) t w\left(f_{j}\right) \rho\left(\alpha\left(f_{j}\right)\right) \\
(1-u) t w\left(f_{j}^{-1}\right) \rho\left(\alpha\left(f_{j}^{-1}\right)\right) & \mathbf{I}_{d}
\end{array}\right]\right) \\
\quad=\left(1-(1-u)^{2} t^{2} w\left(f_{j}\right) w\left(f_{j}^{-1}\right)\right)^{d} \neq 0\left(1 \leqslant j \leqslant m_{1}\right) .
\end{gathered}
$$

Thus, $\mathbf{I}_{m d}+(1-u) t \mathbf{U}_{d} \mathbf{J}_{\rho}$ is invertible. Therefore,

$$
\begin{aligned}
& \operatorname{det}\left(\mathbf{I}_{m d}-t \mathbf{U}_{d}\left(\mathbf{B}_{\rho}-(1-u) \mathbf{J}_{\rho}\right)\right) \\
= & \operatorname{det}\left(\mathbf{I}_{m d}-t \mathbf{U}_{d} \mathbf{L}^{t} \mathbf{K}\left(\mathbf{I}_{m d}+(1-u) t \mathbf{U}_{d} \mathbf{J}_{\rho}\right)^{-1}\right) \operatorname{det}\left(\mathbf{I}_{m d}+(1-u) t \mathbf{U}_{d} \mathbf{J}_{\rho}\right) .
\end{aligned}
$$

By (2), we have

$$
\begin{aligned}
& \operatorname{det}\left(\mathbf{I}_{m d}-t \mathbf{U}_{d}\left(\mathbf{B}_{\rho}-(1-u) \mathbf{J}_{\rho}\right)\right) \\
= & \operatorname{det}\left(\mathbf{I}_{n d}-t^{t} \mathbf{K}\left(\mathbf{I}_{n d}+(1-u) t \mathbf{U}_{d} \mathbf{J}_{\rho}\right)^{-1} \mathbf{U}_{d} \mathbf{L}\right) \operatorname{det}\left(\mathbf{I}_{m d}+(1-u) t \mathbf{U}_{d} \mathbf{J}_{\rho}\right) .
\end{aligned}
$$

Next, we have

$$
\operatorname{det}\left(\mathbf{I}_{m d}+(1-u) t \mathbf{U}_{d} \mathbf{J}_{\rho}\right)=\prod_{i=1}^{m_{1}}\left(1-w\left(f_{i}\right) w\left(f_{i}^{-1}\right)(1-u)^{2} t^{2}\right)^{d} .
$$

Furthermore, the $m d \times n d$ matrix $\mathbf{U}_{d} \mathbf{L}=\left(c_{e v}\right)_{e \in A(D) ; v \in V(D)}$ is given as follows:

$$
c_{e v}:= \begin{cases}w(e) \rho(\alpha(e)) & \text { if } t(e)=v \\ 0 & \text { otherwise }\end{cases}
$$


But, we have

$$
\begin{gathered}
\left(\mathbf{I}_{m d}+(1-u) t \mathbf{U}_{d} \mathbf{J}_{\rho}\right)^{-1} \\
1 / x_{j} \mathbf{I}_{d} \\
=\mathbf{I}_{m_{0} d} \oplus\left(\oplus_{j=1}^{m_{1}}\left[\begin{array}{cc}
-1-u) t w\left(f_{j}\right) / x_{j} \rho\left(\alpha\left(f_{j}\right)\right) \\
-(1-u) t w\left(f_{j}^{-1}\right) / x_{j} \rho\left(\alpha\left(f_{j}^{-1}\right)\right)
\end{array}\right]\right) .
\end{gathered}
$$

where $x_{i}=1-w\left(f_{i}\right) w\left(f_{i}^{-1}\right)(1-u)^{2} t^{2}\left(1 \leqslant i \leqslant m_{1}\right)$.

But, for a symmetric arc $(x, y) \in A(D)$,

$$
\left({ }^{t} \mathbf{K}\left(\mathbf{I}_{m d}+(1-u) t \mathbf{U}_{d} \mathbf{J}_{\rho}\right)^{-1} \mathbf{U}_{d} \mathbf{L}\right)_{x y}=w(x, y) /\left(1-w(x, y) w(y, x)(1-u)^{2} t^{2}\right) \rho(\alpha(x, y)) .
$$

For a nonsymmetric arc $(x, y) \in A(D)$,

$$
\left({ }^{t} \mathbf{K}\left(\mathbf{I}_{m d}+(1-u) t \mathbf{U}_{d} \mathbf{J}_{\rho}\right)^{-1} \mathbf{U}_{d} \mathbf{L}\right)_{x y}=w(x, y) \rho(\alpha(x, y)) .
$$

Furthermore, if $x=y$, then

$$
\left({ }^{t} \mathbf{K}\left(\mathbf{I}_{m d}+(1-u) t \mathbf{U}_{d} \mathbf{J}_{\rho}\right)^{-1} \mathbf{U}_{d} \mathbf{L}\right)_{x x}=-\sum_{o(e)=x, e^{-1} \in A(D)} \frac{(1-u) t w(e) w\left(e^{-1}\right)}{1-w(e) w\left(e^{-1}\right)(1-u)^{2} t^{2}} \mathbf{I}_{d}
$$

Thus,

$$
\begin{gathered}
\operatorname{det}\left(\mathbf{I}_{n d}-t^{t} \mathbf{K}\left(\mathbf{I}_{n d}+(1-u) t \mathbf{U}_{d} \mathbf{J}_{\rho}\right)^{-1} \mathbf{U}_{d} \mathbf{L}\right) \\
=\operatorname{det}\left(\mathbf{I}_{n d}+(1-u) t^{2} \tilde{\mathbf{D}}(D) \bigotimes \mathbf{I}_{d}-t \sum_{g \in \Gamma} \mathbf{A}_{1, g} \bigotimes \rho(g)-t \sum_{g \in \Gamma} \mathbf{A}_{0, g} \bigotimes \rho(g)\right),
\end{gathered}
$$

Therefore, it follows that

$$
\begin{gathered}
\zeta_{D}(w, u, t, \rho, \alpha)^{-1}=\prod_{i=1}^{m_{1}}\left(1-w\left(f_{i}\right) w\left(f_{i}^{-1}\right)(1-u)^{2} t^{2}\right)^{d} \\
\times \operatorname{det}\left(\mathbf{I}_{n d}+(1-u) t^{2} \mathbf{I}_{d} \bigotimes \tilde{\mathbf{D}}(D)-t \sum_{g \in \Gamma} \rho(g) \bigotimes \mathbf{A}_{1, g}-t \sum_{g \in \Gamma} \rho(g) \bigotimes \mathbf{A}_{0, g}\right),
\end{gathered}
$$

By Theorems 6,8, the following result holds.

Corollary 2 (Choe, Kwak, Park and Sato). Let $D$ be a connected digraph, $\Gamma$ a finite group, $\alpha: A(D) \longrightarrow \Gamma$ a pseudo ordinary voltage assignment and $\mathbf{W}=\mathbf{W}(D)$ a weighted matrix of $D$. Then we have

$$
\zeta\left(D^{\alpha}, \tilde{w}, u, t\right)=\prod_{\rho} \zeta_{D}(w, u, t, \rho, \alpha)^{\operatorname{deg} \rho}
$$

where $\rho$ runs over all inequivalent irreducible representations of $\Gamma$. 


\section{Bartholdi edge zeta function of a digraph}

Let $D$ be a connected digraph with $m$ arcs $e_{1}, \ldots, e_{m}$. Furthermore, let $z_{1}, \ldots z_{m}$ be $m$ variables. Set $z_{e_{i}}=z_{i}(1 \leqslant i \leqslant m)$ and $\mathbf{z}=\left(z_{1}, \ldots, z_{m}\right)$. Then the Bartholdi edge zeta function $\zeta(D, w, u)$ of $D$ is defined by

$$
\zeta(D, \mathbf{z}, u)=\prod_{[C]}\left(1-g(C) u^{c b c(C)}\right)^{-1}
$$

where $[C]$ runs over all equivalence classes of prime cycles of $D$. If $D=D_{G}$ is the symmetric digraph of a graph $G$, then the Bartholdi edge zeta function $\zeta\left(D_{G}, \mathbf{z}, u\right)$ of $D_{G}$ is called the Bartholdi edge zeta function $\zeta(G, \mathbf{z}, u)$ of $G$.

Now, set $|V(D)|=n$. Then we define an $n \times n$ matrix $\mathbf{A}_{1}^{\prime}=\mathbf{A}_{1}^{\prime}(D)=\left(a_{x y}\right)$ as follows:

$$
a_{x y}= \begin{cases}z_{(x, y)} /\left(1-z_{(x, y)} z_{(y, x)}(1-u)^{2}\right) & \text { if both }(x, y) \text { and }(y, x) \in A(D), \\ 0 & \text { otherwise. }\end{cases}
$$

Furthermore, an $n \times n$ matrix $\mathbf{D}^{\prime}=\mathbf{D}^{\prime}(D)=\left(d_{x y}\right)$ is the diagonal matrix defined by

$$
d_{x x}=\sum_{o(e)=x, e^{-1} \in A(D)} \frac{z_{e} z_{e^{-1}}}{1-z_{e} z_{e^{-1}}(1-u)^{2}} .
$$

Substituting $t=1$ in Theorem 5 , we obtain the following result.

Corollary 3. Let $D$ be a connected digraph with $m$ arcs and let $\mathbf{z}=\left(z_{1}, \ldots, z_{m}\right)$ be $m$ variables. Then the reciprocal of the Bartholdi edge zeta function of $D$ is given by

$$
\zeta(D, \mathbf{z}, u)^{-1}=\operatorname{det}\left(\mathbf{I}_{n}+(1-u) \mathbf{D}^{\prime}-\mathbf{A}_{1}^{\prime}(D)-\tilde{\mathbf{A}}_{0}\right) \prod_{i=1}^{m_{1}}\left(1-z_{f_{i}} z_{f_{i}^{-1}}(1-u)^{2}\right)
$$

where $n=|V(D)|$ and $f_{1}^{ \pm 1}, \ldots, f_{m_{1}}^{ \pm 1}$ are symmetric arcs of $D$.

If $D=D_{G}$, then

Corollary 4. Let $G$ be a connected graph with $m$ edges and let $\mathbf{z}=\left(z_{1}, \ldots, z_{2 m}\right)$ be $2 m$ variables. and let $\mathbf{W}=\mathbf{W}(G)$ be a weighted matrix of $G$. Then the reciprocal of the Bartholdi edge zeta function of $G$ is given by

$$
\zeta(G, \mathbf{z}, u)^{-1}=\operatorname{det}\left(\mathbf{I}_{n}+(1-u) \mathbf{D}^{\prime}-\mathbf{A}_{1}^{\prime}(G)-\tilde{\mathbf{A}}_{0}\right) \prod_{i=1}^{m}\left(1-z_{f_{i}} z_{f_{i}^{-1}}(1-u)^{2}\right),
$$

where $n=|V(G)|$ and $D(G)=\left\{f_{1}^{ \pm 1}, \ldots, f_{m}^{ \pm 1}\right\}$. 


\section{Example}

Finally, we give an example. Let $D$ be the digraph with three vertices $v_{1}, v_{2}, v_{3}$ and five $\operatorname{arcs}\left(v_{1}, v_{2}\right),\left(v_{2}, v_{1}\right),\left(v_{2}, v_{3}\right),\left(v_{3}, v_{2}\right),\left(v_{3}, v_{1}\right)$. Furthermore, let

$$
\mathbf{W}(D)=\left[\begin{array}{lll}
0 & a & 0 \\
b & 0 & c \\
d & e & 0
\end{array}\right] .
$$

Then we have $n=3, m=5, m_{1}=2$. By Theorem 5 , we have

$$
\begin{aligned}
& \zeta(D, w, u, t)^{-1} \\
= & \left(1-a b(1-u)^{2} t^{2}\right)\left(1-c e(1-u)^{2} t^{2}\right) \operatorname{det}\left(\mathbf{I}_{3}-t \tilde{\mathbf{A}}_{1}-t \tilde{\mathbf{A}}_{0}+(1-u) t^{2} \tilde{\mathbf{D}}\right) \\
= & A B \operatorname{det}\left(\left[\begin{array}{ccc}
1+a b F / A & -a t / A & 0 \\
-b t / A & 1+a b F / A+c e F / B & -c t / B \\
-d t & -e t / B & 1+c e F / B
\end{array}\right]\right) \\
= & 1-(a b+c e) u^{2} t^{2}+a b c e\left(u^{4}-u^{2}\right) t^{4}-a c d t^{3},
\end{aligned}
$$

where $A=1-a b(1-u)^{2} t^{2}, B=1-c e(1-u)^{2} t^{2}$ and $F=(1-u) t^{2}$.

Let $\Gamma=Z_{3}=\left\{1, \tau, \tau^{2}\right\}\left(\tau^{3}=1\right)$ be the cyclic group of order 3 , and let $\alpha: A(D) \longrightarrow Z_{3}$ be the pseudo ordinary voltage assignment such that $\alpha\left(v_{1}, v_{2}\right)=\tau, \alpha\left(v_{2}, v_{1}\right)=\tau^{2}$ and $\alpha\left(v_{2}, v_{3}\right)=\alpha\left(v_{3}, v_{2}\right)=\alpha\left(v_{3}, v_{1}\right)=1$. The characters of $\mathbf{Z}_{3}$ are given as follows: $\chi_{i}\left(\tau^{j}\right)=$ $\left(\xi^{i}\right)^{j}, 0 \leqslant i, j \leqslant 2$, where $\xi=\frac{-1+\sqrt{-3}}{2}$.

Now, we present the weighted Bartholdi $L$-function $\zeta_{D}\left(w, u, t, \chi_{1}, \alpha\right)$ of $D$ associated with $\chi_{1}$ and $\alpha$. Theorem 8 implies that

$$
\begin{aligned}
\zeta_{D}\left(w, u, t, \chi_{1}, \alpha\right)^{-1} & =A B \operatorname{det}\left(\mathbf{I}_{3}-t \sum_{i=0}^{2} \chi_{1}\left(\tau^{i}\right) \mathbf{A}_{1, \tau^{i}}-t \sum_{i=0}^{2} \chi_{1}\left(\tau^{i}\right) \mathbf{A}_{0, \tau^{i}}+(1-u) t^{2} \tilde{\mathbf{D}}\right) \\
& =A B \operatorname{det}\left(\left[\begin{array}{ccc}
1+a b F / A & -a t \xi / A & 0 \\
-b t \xi^{2} / A & 1+a b F / A+c e F / B & -c t / B \\
-d t & -e t / B & 1+c e F / B
\end{array}\right]\right) \\
& =1-(a b+c e) u^{2} t^{2}+a b c e\left(u^{4}-u^{2}\right) t^{4}-a c d t^{3} \xi .
\end{aligned}
$$

Similarly, we have

$$
\zeta_{D}\left(w, u, t, \chi_{2}, \alpha\right)^{-1}=1-(a b+c e) u^{2} t^{2}+a b c e\left(u^{4}-u^{2}\right) t^{4}-a c d t^{3} \xi^{2} .
$$

By Corollary 2, it follows that

$$
\begin{aligned}
\zeta\left(D^{\alpha}, \tilde{w}, u, t\right)^{-1} & =\zeta(D, w, u, t)^{-1} \zeta_{D}\left(w, u, t, \chi_{1}, \alpha\right)^{-1} \zeta_{D}\left(w, u, t, \chi_{2}, \alpha\right)^{-1} \\
& =\left(1-(a b+c e) u^{2} t^{2}+a b c e\left(u^{4}-u^{2}\right) t^{4}\right)^{3}-a^{3} c^{3} d^{3} t^{9}
\end{aligned}
$$


If $w\left(e^{-1}\right)=w(e)^{-1}$ for each symmetric arc $e \in A(D)$, then

$$
\begin{gathered}
\zeta(D, w, u, t)^{-1}=1-2 u^{2} t^{2}+\left(u^{4}-u^{2}\right) t^{4}-a c d t^{3} \\
\zeta_{D}\left(w, u, t, \chi_{i}, \alpha\right)^{-1}=1-2 u^{2} t^{2}+\left(u^{4}-u^{2}\right) t^{4}-a c d t^{3} \xi^{i}(i=1,2)
\end{gathered}
$$

and

$$
\zeta\left(D^{\alpha}, \tilde{w}, u, t\right)^{-1}=\left(1-2 u^{2} t^{2}+\left(u^{4}-u^{2}\right) t^{4}\right)^{3}-a^{3} c^{3} d^{3} t^{9} .
$$

\section{Acknowledgment}

We would like to thank the referee for many valuable comments and helpful suggestions.

\section{References}

[1] L. Bartholdi, Counting paths in graphs, Enseign. Math. 45 (1999) 83-131.

[2] H. Bass, The Ihara-Selberg zeta function of a tree lattice, Internat. J. Math. 3 (1992) 717-797.

[3] Y. Choe, J. H. Kwak, Y. S. Park and I. Sato, Bartholdi zeta and $L$-functions of weighted digraphs, their coverings and products, Adv. Math. 213 (2007), 865-886.

[4] D. Foata and D. Zeilberger, A combinatorial proof of Bass's evaluations of the IharaSelberg zeta function for graphs, Trans. Amer. Math. Soc. 351 (1999) 2257-2274.

[5] J. L. Gross and T. W. Tucker, Topological Graph Theory, (Wiley, New York, 1987).

[6] K. Hashimoto, Zeta Functions of Finite Graphs and Representations of $p$-Adic Groups, in Adv. Stud. Pure Math. 15 (Academic Press, New York, 1989) 211-280.

[7] Y. Ihara, On discrete subgroups of the two by two projective linear group over $p$-adic fields, J. Math. Soc. Japan 18 (1966) 219-235.

[8] M. Kotani and T. Sunada, Zeta functions of finite graphs, J. Math. Sci. U. Tokyo 7 (2000) 7-25.

[9] H. Mizuno and I. Sato, Bartholdi zeta functions of graph coverings, J. Combin. Theory Ser. B. 89 (2003) 27-41.

[10] J.-P. Serre, Linear Representations of Finite Groups, (Springer-Verlag, New York, 1977).

[11] H. M. Stark and A. A. Terras, Zeta functions of finite graphs and coverings, Adv. Math. 121 (1996) 124-165.

[12] T. Sunada, L-Functions in Geometry and Some Applications, in Lecture Notes in Math. 1201 (Springer-Verlag, New York, 1986) 266-284.

[13] T. Sunada, Fundamental Groups and Laplacians (in Japanese), (Kinokuniya, Tokyo, 1988).

[14] Y. Watanabe and K. Fukumizu, Graph zeta function in the Bethe free energy and loopy belief propagation, Adv. Neural Inform. Proc. Sys. 22 (2010), 2017-2025. 